\title{
Essential genes and miRNA-mRNA network contributing to the pathogenesis of idiopathic pulmonary arterial hypertension
}

\section{Shengyu Hao}

Zhongshan Hospital Fudan University https://orcid.org/0000-0003-3162-5460

\section{Pan Jiang}

Department of Respiratory Medicine, Zhongshan Hospital affiliated to Fudan University, Shanghai, 200032, China

\section{Liang Xie}

Department of Respiratory Medicine, Zhongshan Hospital affiliated to Fudan University, Shanghai, 200032, China

\section{Guiling Xiang}

Department of Respiratory Medicine, Zhongshan Hospital affiliated to Fudan University, Shanghai, 200032, China

\section{Zilong Liu}

Department of Respiratory Medicine, Zhongshan Hospital affiliated to Fudan University, Shanghai, 200032, China

\section{Weiping Hu}

Department of Respiratory Medicine, Zhongshan Hospital affiliated to Fudan University, Shanghai, 200032, China

\section{Qinhan Wu}

Department of Respiratory Medicine, Zhongshan Hospital affiliated to Fudan University, Shanghai, 200032, China

\section{Shanqun Li (Dzslsq1966@163.com )}

Department of Respiratory Medicine, Zhongshan Hospital affiliated to Fudan University, Shanghai, 200032, China

\section{Research}

Keywords: idiopathic pulmonary arterial hypertension, hub genes, lung tissues, miRNA

Posted Date: October 12th, 2020

DOI: https://doi.org/10.21203/rs.3.rs-89957/v1

License: (c) (i) This work is licensed under a Creative Commons Attribution 4.0 International License. Read Full License

Version of Record: A version of this preprint was published at Frontiers in Cardiovascular Medicine on May 5th, 2021. See the published version at https://doi.org/10.3389/fcvm.2021.627873. 


\section{Abstract}

Background: Idiopathic pulmonary arterial hypertension (IPAH) is a life-threatening disease. Owing to its high fatality rate and narrow therapeutic options, identification of the pathogenic mechanisms of IPAH is becoming increasingly important.

Methods: In our research, we utilized the Robust Rank Aggregating (RRA) method to integrate four eligible PAH microarray datasets and identified the significant differentially expressed genes (DEGs) between IPAH and normal samples. Gene Ontology (GO) and Kyoto Encyclopedia of Genes and Genomes (KEGG) pathways were performed to analyze their functions. The interaction network of protein-protein internet (PPI) was constructed to explore the correlation between these DEGs. The functional modules and hub genes were further identified by the weighted gene coexpression network analysis (WGCNA). Moreover, a miRNA microarray dataset was involved and analyzed to filter differentially expressed miRNA (DE-miRNAs). Potential target genes of screened DE-miRNAs were predicted and merged with DEGs to explore a miRNA-mRNA network in IPAH. Some hub genes were selected and validated by RT-PCR in lung tissues from PAH animal model.

Results: A total of 260 DEGs, consisting of 183 upregulated and 77 down-regulated significant DEGs were identified, and some of those genes were novel. Their molecular roles in the etiology of IPAH remained vague. The most crucial functional module involved in IPAH mainly enriched in biological processes, including leukocyte migration, cell chemotaxis, and myeloid leukocyte migration. Construction and analysis of the PPI network showed that CXCL10, CXCL9, CCR1, CX3CR1, CX3CL1, CXCR2, CXCR1, PF4, CCL4L1, and ADORA3 were recognized as top10 hub genes with high connectivity degrees. WGCNA further identified five main functional modules involved in the pathogenesis of IPAH. 12 upregulated DE-miRNAs and 9 downregulated DE-miRNAs were identified. Among them, four downregulated DEGs, and eight upregulated DEGs were supposed to be negatively regulated by three upregulated DE-miRNAs, and three downregulated DE-miRNAs, respectively.

Conclusions: This study identifies some key and functional coexpression modules involved in IPAH, as well as a potential IPAHrelated miRNA-mRNA regulated network. It provides deepening insights into the molecular mechanisms and provides vital clues in seeking novel therapeutic targets for IPAH.

\section{Background}

PAH is a progressive and fatal disease characterized by abnormal cellular apoptotic resistance and vascular remodeling leading to elevated pulmonary pressures and, eventually, right heart failure[1]. According to the previous studies, the average time from symptom onset to diagnosis is about two years, and the mean survival time of untreated PAH patients is 2.8 years[2]. PAH is classified into five major groups, and IPAH counts for about $40 \%$ of cases. It is considered that PAH develops in response to various genetic abnormalities and is triggered by environmental risks[2]. Currently, the approved treatments for PAH have been reported to successfully target vasoconstrictive and proliferative mediators, such as prostacyclin receptor agonists, endothelin receptor antagonists, soluble cGMP stimulators and phosphodiesterase type- 5 inhibitors, leading to improved exercise capacity and clinical prognosis[3]. However, the individual response to therapy is not uniform, and the patient's condition may be improved, but some worsen[4-6]. As a result, clarifying the gene-specific expression helps explore the pathogenic mechanisms or seek treatment for IPAH.

In the past few decades, microarray analysis has been used for gene-wide expression profiling in lung tissues or peripheral blood from PAH patients or experimental animals to define PAH's pathobiology[6-10]. However, the findings are inconsistent between different researches, which may be caused by various analysis platforms, limited sample sizes, different methods, no classification of PAH, and not for IPAH. Although Elinoff et al. performed an integrated analysis of blood expression profiles in PAH[8], the microarray datasets from the lung tissues may be better and more directly reflect the PAH's pathological process. The RRA method has been used to combine available datasets from independent researches and perform integrative analysis to improve the statistical power and reliability of results[11].

In this study, we aimed to characterize key genes, pathways, and miRNA-mRNA networks related to IPAH. Using the RRA method, we integrated four pulmonary microarray datasets of IPAH patients to identify the character gene expression for IPAH. The pathway enrichment analysis of GO and KEGG, and module alteration in patients with IPAH were conducted to screen hub 
genes. Furthermore, we utilized a miRNA dataset to identify the possible network of mRNA-miRNA in IPAH. Several hug genes were randomly selected and subsequently verified by RT-PCR in a widely used mice model of PAH.

\section{Methods}

\subsection{Microarray datasets}

Two common databases, ArrayExpress (https://www.ebi.ac.uk/arrayexpress/) and Gene Expression Omnibus (GEO) (https://www.ncbi.nlm.nih.gov/geo/) were used to search datasets of IPAH patients. We used the following search terms: pulmonary hypertension or pulmonary arterial hypertension or PAH or PH or idiopathic pulmonary arterial hypertension or IPAH. Two researchers independently screened the two databases and selected relevant datasets based on the same following criteria:

1. The genome-wide gene or non-coding RNA profiling of IPAH patients and controls were involved in the datasets detected by a high-throughput array or next-generation sequencing;

2. Samples were from human lung tissues;

3. Data that could be used for re-analyzation, such as raw data or processed data;

4. Datasets not meeting the above criteria were excluded.

Our workflow for bioinformatics analysis of publicly available datasets is illustrated in Fig. 1.

\subsection{RRA analysis and integration of datasets}

The gene expression profiling was annotated through the corresponding annotation package and the R software (version 3.6.3). The expression data were further normalized by using the "limma" package (Supplementary Fig. 1). Then, we performed RRA analysis to rank the up-regulated and down-regulated expression genes through the R package of "Robust Rank Aggregation" and listed the genes according to their fold changes[12]. In the final list, a Bonferroni correction was performed to cut down the false-positive results, and $p$ values that represented the possibility of ranking high were calculated for each gene.

\subsection{Functional enrichment analysis and PPI analysis}

We performed GO and KEGG pathway enrichment analysis by using the cluster profile function of R software to match the biological themes of the gene clusters, with a cut-off $p$-value of 0.05 . The STRING database (https://string-db.org/cgi/input.pl) was used to establish a PPI network. In the network, the significant Molecular Complex Detection (MCODE) and hub genes were present by using Cytoscape software (version 3.8.0).

\subsection{WGCNA}

To perform WGCNA analysis, genes with both $p$-value $<0.05$ and logarithmic fold changes (logFCs) $>0.25$ were selected from RRA results. To improve the number of samples and avoid generating less reliable results, we integrated and normalized the four datasets by batch normalization using the "sva" and "limma" package in the R computing environment. We transformed the adjacency matrix into a topological overlap matrix (TOM) and parted the genes into different modules based on the TOM-based dissimilarity measure. Key modules were identified by setting the soft-thresholding power of 6 (scale-free $\mathrm{R}^{2}=0.9$ ), cut height of 0.25 , and minimal module size of 20 . We further set gene significance (GS) $>0.3$ and module membership (MM) $>0.8$ to define the hub genes in WGCNA analysis.

\subsection{Identification of DE-miRNAs and prediction of target genes}

We downloaded the microRNA expression dataset GSE67597 and identified pulmonary DE-miRNAs in IPAH patients by using the GEO2R online tool (https://www.ncbi.nlm.nih.gov/geo/geo2r/) with the thresholds of both $p<0.05$ and $|\log 2 \mathrm{FC}|>2$. The miRNet database, a useful tool for miRNAs analysis, was used to predict the target genes of DE-miRNAs[13]. To visualize the relationship between DE-miRNAs and predicted target mRNAs, we established a miRNA-mRNA network by using Cytoscape (version 3.8.0).

\subsection{Animal models of PAH and hemodynamic measurements}


In the experimental group, 8-10-week-old male mice weekly received a subcutaneous injection of $20 \mathrm{mg} / \mathrm{kg}$ SU5416 (an angiogenesis inhibitor), which was dissolved in carboxymethylcellulose solution. They were cultured in a chamber under hypoxia $\left(10 \% \mathrm{O}_{2}\right)$ for 28 days. Mice in the control group were exposed to normoxia, injected with an equivalent volume of the dissolvent solution according to their weights. Two groups were provided with food and water ad libitum. At the end of the experiment, we used isoflurane to anesthetize the mice and measured their right ventricular systolic pressure (RVSP) by using a Millar pressure transducer catheter through right-sided heart catheterization, as we previously described[14]. To evaluate the RV remodeling, we weighed the wall of $R V$ and the left ventricle plus septum $(L V+S)$ to calculate the ratio of $(R V / L V+S)$.

\subsection{Immunohistochemistry, immunofluorescence, and imaging}

The whole heart and left lung were embedded in paraffin, sliced into $5 \mu \mathrm{m}$ sections, and then were stained for hematoxylin and eosin (H\&E), Masson, and immunofluorescence followed by examination with a light microscope (Nikon).

\subsection{Real-time polymerase chain reaction (RT-PCR)}

Total RNA from lungs was isolated using Trizol, and the extracted RNA was reverse transcribed into complementary DNA (cDNA) using the PrimeScript RT reagent kit (Takara Bio). RT-PCR was carried out using SYBR Green Premix Ex Taq (Takara Bio) to detect the expressions of the genes, which were selected from our analysis. The primer sequences were provided in supplements.

\subsection{Statistical Analysis}

we used R software and GraphPad Prism 7 to perform statistical analyses, presented data as mean \pm standard error, and calculated statistical significance by the Student $t$-test. $p$ values $<0.05$ was considered statistically significant.

\section{Results}

\subsection{PAH microarray datasets}

After filtering ArrayExpress and GEO based on the eligibility criteria, five microarray datasets of PH were finally selected. The information of these datasets was listed, including study country, types of RNA source, GEO accession ID, detection platforms, experiment type, and sample information. The number of IPAH patients in each study ranged from 6 to 32 , and the controls ranged from 8 to 25. Finally, a total of 64 IPAH patients and 58 controls were involved in our study (Table 1). 
Table 1

Summary of those 5 expression datasets included in the analysis.

\begin{tabular}{|c|c|c|c|c|c|c|c|c|c|c|}
\hline Study & Country & $\begin{array}{l}\text { Source } \\
\text { types }\end{array}$ & Platform & $\begin{array}{l}\text { GSE } \\
\text { Accesion }\end{array}$ & $\begin{array}{l}\text { Experiment } \\
\text { type }\end{array}$ & $\begin{array}{l}\text { Total } \\
\text { Number }\end{array}$ & Control & IPAH & PAH & Others \\
\hline $\begin{array}{l}\text { Feghali- } \\
\text { Bostwick } \\
2013\end{array}$ & USA & $\begin{array}{l}\text { Lung } \\
\text { tissues }\end{array}$ & GPL16221 & GSE48149 & mRNA & 53 & 9 & 8 & 8 & $\begin{array}{l}\text { SSC- } \\
\text { PAH: } \\
10\end{array}$ \\
\hline $\begin{array}{l}\text { Mura } \\
2018\end{array}$ & Canada & $\begin{array}{l}\text { Lung } \\
\text { tissues }\end{array}$ & GPL6244 & GSE113439 & mRNA & 26 & 11 & 6 & 15 & $\begin{array}{l}\text { CTD- } \\
\text { PAH: } 4 \text {; } \\
\text { CHD- } \\
\text { PAH: } 4 ; \\
\text { CTEPH } \\
1\end{array}$ \\
\hline $\begin{array}{l}\text { Stearman } \\
2018\end{array}$ & USA & $\begin{array}{l}\text { Lung } \\
\text { tissues }\end{array}$ & GPL6244 & GSE117261 & mRNA & 83 & 25 & 32 & 58 & $\begin{array}{l}\text { APAH: } \\
17 \\
\text { FPAH: } \\
5 ; \\
\text { Other: } \\
3 ; \\
\text { WHO4: } \\
1\end{array}$ \\
\hline $\begin{array}{l}\text { Ferhaan } \\
2019\end{array}$ & USA & $\begin{array}{l}\text { Lung } \\
\text { tissues }\end{array}$ & GPL6480 & GSE15197 & mRNA & 39 & 13 & 18 & 26 & $\begin{array}{l}\text { IPF-PH: } \\
8\end{array}$ \\
\hline Gao 2017 & USA & $\begin{array}{l}\text { Lung } \\
\text { tissues }\end{array}$ & GPL18402 & GSE67597 & microRNA & 15 & 8 & 7 & 7 & None \\
\hline
\end{tabular}

Note. PAH, pulmonary arterial hypertension; IPAH, idiopathic PAH; ssc-PAH, SSc-associated PAH; IPF, idiopathic pulmonary fibrosis; IPAH, idiopathic PAH; FPAH, family PAH; CTD-PAH, PAH secondary to connective tissue disease; $C H D-P A H, P A H$ secondary to congenital heart disease; CTEPH, chronic thromboembolic pulmonary hypertension

\subsection{Identification of robust DEGs by the RRA method}

A total of 183 up-regulated and 77 down-regulated DEGs were successfully identified by integrating four genome-wide gene expression datasets through the RRA method (Supplementary Table 1). Among the up-regulated genes, PKP2 was ranked as the first one ( $p$-value $=4.71 \mathrm{E}-07$, adjusted $p$-value $=1.36 \mathrm{E}-02)$, followed by ALAS2 ( $p$-value $=1.11 \mathrm{E}-06$, adjusted $p$-value $=3.19 \mathrm{E}-02)$. Meanwhile, RNASE2 ( $p$-value $=8.94 \mathrm{E}-10$, adjusted $p$-value $=2.57 \mathrm{E}-05)$ and PROK2 ( $p$-value $=2.20 \mathrm{E}-08$, adjusted $p$-value $=6.33 \mathrm{E}$ 04) were ranked as the first and the second down-regulated genes in RRA analysis. The top 30 up-regulated and down-regulated genes in IPAH were illustrated by a heatmap (Fig. 2). Among these genes, the roles of some ones had been well explored in PAH, such as interleukin 13 receptor alpha 2 (IL13RA2), angiotensin I converting enzyme 2 (ACE2)[15] and vascular cell adhesion molecule 1 (VCAM1)[16]. Notably, some genes were novel, and their functions in PAH had not been researched in published literature, such as hemoglobin alpha 2 (HBA2), frizzled-related protein 2 (SFRP2), and ribonucleases 2 (RNASE2).

\subsection{Functional enrichment analysis of DEGs}

To research the potential biological pathways involved in IPAH, we performed GO and KEGG to analyze the DEGs. Several enrichment biological processes in $\mathrm{GO}$ terms were identified, such as leukocyte migration, cell chemotaxis, and myeloid leukocyte chemotaxis (Fig. 3A). In terms of molecular function, receptor-ligand activity was listed as the most significantly GO terms (Fig. 3B). Moreover, some cellular component terms were enriched, such as external side of plasma membrane and collagen-containing extracellular matrix (Fig. 3C). According to KEGG analysis, we found that the DEGs were mostly associated with cytokine-cytokine receptor interaction, chemokine signaling pathway, viral protein interaction with cytokine and cytokine receptor, fluid shear stress and atherosclerosis, and IL-17 signaling pathway (Fig. 3D).

Next, PPI network of these DEGs was constructed by using the STRING database. The visualization was carried out using Cytoscape software (Fig. 4A). MCODE plugin used to find the top hub genes, the top 3 closely connected modules were identified 
(Fig. 4B-D and Supplementary Table 2).

\subsection{WGCNA}

To further identify the functional and meaningful modules most associated with IPAH, we utilized WGCNA analysis in these four datasets (Fig. 5A). We selected genes with $p \otimes 0.05$ and logFCs $₫ 0.25$ from the ranked gene list to cover enough genes in WGCNA analysis. Finally, 14 modules were identified by setting the soft-thresholding power as 6 (scale-free $\mathrm{R}^{2}=0.9$ ) and cut height as 0.25 (Fig. 5B-D; non-clustering DEGs shown in gray). The correlations between module and IPAH were illustrated in a heatmap, and the midnight-blue module was found to be the most highly correlated module with IPAH (Fig. 5E-F). The midnight-blue module contained 42 genes shown in Fig. $5 \mathrm{G}$ (correlation coefficient $=0.49, p=1 \mathrm{E}-08 \mathrm{G}$ ). We selected top20 hub genes from the midnight-blue module: CXCL10, IFNG, CCL3, CXCL9, VCAM1, ANKRD22, IL13, SAA1, ITGAMT, TNFSF8, NCR1, EOMES, KLRC3, SLC14A1, ADORA3, ZAP70, CD96, CCL5, LCK, and BTLA. Venn diagram displayed the overlap of genes between PPI analysis and WGCNA analysis. The results showed that seven hub genes (INFG, IL13, CXCL10, SAA1, CCL3, CXCL9, and ADORA3) were found in the top20 hub genes of PPI and top20 genes of the midnight-blue module (Fig. 5H).

\subsection{The potential miRNA-mRNA regulatory network}

It is widely acknowledged that miRNA exerts an essential effect in PAH by negatively regulating target $m R N A$. Therefore, we utilized GEO2R tool to identify the DE-miRNAs in IPAH and predicted the downstream target genes of DE-miRNAs by using miRNet database. Eventually, we predicted 1816 and 773 target genes for the 13 up-regulated DE-miRNAs and nine downregulated DE-miRNAs, respectively. Furthermore, the up-regulated DE-miRNA-target gene network and down-regulated DE-miRNAtarget gene network were established and presented (Fig. 6A and D). The number of target genes for each DE-miRNA was listed in Fig. 6B and E. The common genes in DEGs and predicted target genes were identified and shown by the Venn diagram (Fig. 6C and F). As a consequence, we found that four target genes (LILRA2, RPS4Y1, SFN, and VENTX) were negatively regulated by increased miRNA-500a-3p, miRNA-31-5p or miRNA-6074; and eight up-regulated genes (SLFN5, ANKRD50, XIAP, SYTL3, PDE4D, VCAM1, FGD4, and SECISBP2L) were the target of miRNA-1178-3p, miRNA-302f, or miRNA-495-5p (Fig. 7).

\subsection{Validation through qRT-PCR}

Chronic hypoxia exposure with SU5416 injection $(\mathrm{CH}+\mathrm{SU})$ is a well-studied and published method to make animal models of $\mathrm{PAH}$. However, there are currently no perfect animal models that replicate entirely human PAH. To investigate whether the pathological changes of IPAH in human also occurs in $\mathrm{CH}+\mathrm{SU}$ mice, and to validate the expressions of hub genes found in our analysis, we established PAH model. After a 28-day exposure to $\mathrm{CH}$ with the administration of SU5416, mice had a significant elevation in RVSP (Fig. 8A) and RV/LV + S compared with those in control mice (Fig. 8B and C). Meanwhile, $\mathrm{CH}+\mathrm{SU}$ treatment resulted in pulmonary vascular remodeling, evidenced by the increased media (Fig. 8D-F). Then, we detected the expression of selected genes in our successfully established PAH model. As shown in Fig. 9, the expressions of PKP2 (Fig. 9A) were significantly up-regulated. At the same time, ADORA3, PROK2, IL-13 (Fig. 9B, C and D) were downregulated in the lungs from $\mathrm{CH}$ + SU mice, which were consistent with the results in the datasets of IPAH patients. Meanwhile, we found inconsistent gene expression between the animal model and IPAH patients; for example, CXCL10 (Fig. 9E) was downregulated, while SFN (Fig. 9F) was up-regulated in $\mathrm{CH}+\mathrm{SU}$ mice, which were contrary to the findings in IPAH patients. The expression of SFRP2 showed no difference between normoxic and $\mathrm{CH}+\mathrm{SU}$ mice (Fig. 9G), but significantly up-regulated in IPAH patients based on RRA analysis.

\section{Discussion}

Currently, IPAH is still a progressive, deadly, and not curable disease. Drug therapy has improved symptoms and signs of IPAH, but the mortality remains high, with survival rates estimated at $57-75 \%$ at five years. The lack of improvement in survival is at least partially attributable to limitations in understanding the mechanisms of IPAH. Although many researchers attempted performing microarray and RNA-seq to screen novel biomarkers and possible therapeutic targets for IPAH, inconsistent results were observed between different studies. To our knowledge, this is the first work to perform RRA, WGCNA analysis, and miRNA dataset to identify significant hub genes and miRNA-mRNA networks related to IPAH. Although Elinoff et al. recently compared 
gene expression between PAH and control samples for each dataset and obtained DEGs by using R meta-package, the results are based on the blood dataset with no classification of PAH[8]. Tao et al. performed a WGCNA analysis between IPAH and control samples; however, this study only contained one blood dataset with 16 IPAH patients and ten healthy controls[17].To minimize variability, we enrolled lung-tissue datasets from IPAH patients and controls. We involved four qualified PAH datasets into the RRA analysis and observed some significantly up-regulated or down-regulated DEGs, some of which, such as ACE2 and IL-13, have been reported to play a vital role in PAH pathogenesis. Notably, some DEGs found in our research have never been reported in IPAH, such as PKP2, N4BP2, and ANKRD1. PKP2 was reported to target miR-200b, and deficiency of PKP2 impairs cell-cell contacts, particularly in response to mechanical stress or stretch. N4BP2 was reported to be involved in pulmonary fibrosis[18] and nasopharyngeal carcinoma[19].

It is reported that pathogenic mutations have been observed in approximately $25 \%$ of IPAH without a prior family history of the disease. Among them, the bone morphogenetic protein receptor type II (BMPR2) gene is the single most common causal factor for hereditary cases[20]. However, BMPR2 $(p=0.17577$, logFCs $=0.237)$ is not listed in the DEGs in our research. The reason may be that not all carriers of BMPR2 mutations developed PAH and other genetic factors acting as modifiers are needed. Besides, other genes involved in the BMP signaling pathway, such as BMP6, BMP5, SMAD9, and SMAD5 are significantly up-regulated in our study. The contribution of these genes to PAH is less well understood but appears to be connected to the capacity to regulate cell growth and survival in the pulmonary arteries.

The pathogenesis of IPAH is complex and heterogeneous. Consistent with published data, the enrichment of these DEGs in GO terms, such as leukocyte migration[21, 22], collagen-containing extracellular matrix (ECM)[23, 24], cytokine activity[25, 26] and antioxidant activity[27], confirms their involvement in the development of IPAH. Besides, enrichment of the identified DEGs in some KEGG pathways, such as the chemokine signaling pathway[25, 26], IL-17 signaling pathway[28, 29], and NF-kappa B signaling pathway $[30,31]$ also suggests the relevance in IPAH pathogenesis. Based on GO and KEGG analysis results, we suggest that these DEGs are closely associated with immune response and IPAH development. After constructing a coexpression network by PPI and identifying hub genes through WGCNA, we eventually obtained seven hub genes (INFG, IL-13, CXCL10, SAA1, CCL3, CXCL9, ADORA3). Some of them were demonstrated to exert roles in the pathogenesis of PAH.

In the past few years, increasing researches have suggested that expression changes of miRNAs and downstream target genes are closely associated with the development of PAH. In this present study, we conducted an integrated analysis using miRNA from GEO and DEGs from RRA analysis. Three up-regulated DE-miRNAs, and three downregulated DE-miRNAs were finally identified. The miRNA-mRNA network screened in our research has been reported in other pulmonary diseases. For example, hsamir-31-5p (miR-31-5p) is found to be significantly up-regulated in bronchial biopsies from patients with asthma and chronic obstructive pulmonary disease[32], and lung tissues from non-small lung cancer patients[33]. These studies have shown that miR-31-5p mediated cell proliferation, apoptosis, migration, and Warburg effect[34], which were also vital for the development of $\mathrm{PAH}[35]$. Hsa-miR-500a-3p (miR-500a-3p) is another pivotal miRNA in our integrated network and is up-regulated in this study and in the original study[36]. For example, miR-500a-3p is strongly associated with the survival of patients with lung adenocarcinoma and is up-regulated in various human cancers and non-neoplastic diseases, including liver cancer, gastric cancer, and prostate cancer[37-40].

Experimental models of PAH are critical to gaining a better understanding of pathogenesis and to performing preclinical testing of novel therapeutic approaches. To date, the majority of experimental models are animal models, and the $\mathrm{CH}+\mathrm{SU}$ mouse model is one of the most commonly used models for PAH. In our research, genes randomly selected from hug genes were validated by RT-PCR in the lungs of $\mathrm{CH}+\mathrm{SU}$ mice. However, the mRNA expression trends in mice were not wholly consistent with those in IPAH patients. We postulate that this species difference in gene expression may partly explain the inconsistency. Otherwise, the pathological changes of IPAH have not been fully clarified. Animal models can simulate the process of PAH, but unable to be entirely consistent with human IPAH. The difference between mice and IPAH patients reminds us that we should be cautious when drawing conclusions based on experimental animal models. There are several limitations in our study, and future research is needed to validate the findings. First, we did not obtain lung tissues from IPAH patients to perform validation in IPAH patients. Secondly, we did not test the selected genes in other PAH animal models. Finally, assays in vitro and in vivo will be needed to explore the molecular mechanisms and pathways.

Page $7 / 20$ 


\section{Conclusions}

In this study, we characterized some DEGs, their enrichment pathways, significant gene modules, and miRNA-mRNA network in IPAH by comprehensively utilizing RRA, GO, KEGG, PPI, WGCNA, and other bioinformatics tools. These findings in our work provide new and deepening insights into IPAH. Notably, some of these genes, miRNAs, pathways, and networks are novel, and more work still needs to be done to explore their roles in the pathogenesis of IPAH.

\section{Abbreviations}

IPAH

idiopathic pulmonary arterial hypertension

RRA

Robust Rank Aggregating

DEGs

differentially expressed genes

GO

Gene Ontology

KEGG

Kyoto Encyclopedia of Genes and Genomes

PPI

protein-protein internet

WGCNA

weighted gene coexpression network analysis

DE-miRNAs

differentially expressed miRNA

GEO

Gene Expression Omnibus

MCODE

Molecular Complex Detection

logFCs

logarithmic fold changes

TOM

a topological overlap matrix

GS

gene significance

MM

module membership

RVSP

right ventricular systolic pressure

$\mathrm{LV}+\mathrm{S}$

left ventricle plus septum

cDNA

complementary DNA

IL13RA2

13 receptor alpha 2

ACE2

angiotensin I converting enzyme 2

VCAM1

vascular cell adhesion molecule 1 
HBA2

hemoglobin alpha 2

SFRP2

frizzled-related protein 2

RNASE2

ribonucleases 2

$\mathrm{CH}+\mathrm{SU}$

Chronic hypoxia exposure with SU5416 injection

BMPR2

the bone morphogenetic protein receptor type II

$\mathrm{ECM}$

collagen-containing extracellular matrix

\section{Declarations}

\section{Ethics approval and consent to participate}

Not applicable

Consent for publication

Not applicable

\section{Availability of data and materials}

The datasets used and analyzed during the current study are available from the corresponding author with a reasonable request.

\section{Competing interests}

The authors have no conflicts of interest to declare.

\section{Funding}

This work was funded by grants from the National Key Research and Development Program of China (No. 2018YFC1313600) and the National Natural Science Foundation of China (No. 81570081, 81770083).

\section{Authors' Contributions}

S.H. designed and performed the study. P. J., L. X. and G. X. contributed to the literature research. Z. L., Q. H., W. H. and S. L. reviewed and edited the manuscript. All authors read and approved the manuscript.

\section{Acknowledgments}

The authors thank Dan Meng and Qinhan Li for their technical assistance in animal handling.

\section{References}

1. Zolty R. Pulmonary arterial hypertension specific therapy: The old and the new. Pharmacol Ther 2020:107576.

2. "2015 ESC/ERS Guidelines for the diagnosis and treatment of pulmonary hypertension. The Joint Task Force for the Diagnosis and Treatment of Pulmonary Hypertension of the European Society of Cardiology (ESC) and the European Respiratory Society (ERS)." Nazzareno Galie, Marc Humbert, Jean-Luc Vachiery, Simon Gibbs, Irene Lang, Adam Torbicki, Gerald Simonneau, Andrew Peacock, Anton Vonk Noordegraaf, Maurice Beghetti, Ardeschir Ghofrani, Miguel Angel Gomez 
Sanchez, Georg Hansmann, Walter Klepetko, Patrizio Lancellotti, Marco Matucci, Theresa McDonagh, Luc A. Pierard, Pedro T. Trindade, Maurizio Zompatori and Marius Hoeper. Eur Respir J 2015; 46: 903-975. Eur Respir J 2015, 46:1855-1856.

3. JR K, CG E, DJ L, J F-H EBLDKF, JJ SMK, EB R. R, et al: Therapy for Pulmonary Arterial Hypertension in Adults: Update of the CHEST Guideline and Expert Panel Report. 2019, 155:565-586.

4. Rhodes CJ, Wharton J, Ghataorhe P, Watson G, Girerd B, Howard LS, Gibbs JSR, Condliffe R, Elliot CA, Kiely DG, et al. Plasma proteome analysis in patients with pulmonary arterial hypertension: an observational cohort study. Lancet Respir Med. 2017;5:717-26.

5. Graf S, Haimel M, Bleda M, Hadinnapola C, Southgate L, Li W, Hodgson J, Liu B, Salmon RM, Southwood M, et al. Identification of rare sequence variation underlying heritable pulmonary arterial hypertension. Nat Commun. 2018;9:1416.

6. Rhodes CJ, Ghataorhe P, Wharton J, Rue-Albrecht KC, Hadinnapola C, Watson G, Bleda M, Haimel M, Coghlan G, Corris PA, et al. Plasma Metabolomics Implicates Modified Transfer RNAs and Altered Bioenergetics in the Outcomes of Pulmonary Arterial Hypertension. Circulation. 2017;135:460-75.

7. Arwood MJ, Vahabi N, Lteif C, Sharma RK, Machado RF, Duarte JD. Transcriptome-wide analysis associates ID2 expression with combined pre- and post-capillary pulmonary hypertension. Sci Rep. 2019;9:19572.

8. Elinoff JM, Mazer AJ, Cai R, Lu M, Graninger G, Harper B, Ferreyra GA, Sun J, Solomon MA, Danner RL. Meta-analysis of blood genome-wide expression profiling studies in pulmonary arterial hypertension. Am J Physiol Lung Cell Mol Physiol. 2020;318:L98-I111.

9. Saygin D, Tabib T, Bittar HET, Valenzi E, Sembrat J, Chan SY, Rojas M, Lafyatis R. Transcriptional profiling of lung cell populations in idiopathic pulmonary arterial hypertension. Pulm Circ 2020, 10.

10. Stearman RS, Bui QM, Speyer G, Handen A, Cornelius AR, Graham BB, Kim S, Mickler EA, Tuder RM, Chan SY, Geraci MW. Systems Analysis of the Human Pulmonary Arterial Hypertension Lung Transcriptome. Am J Respir Cell Mol Biol. 2019;60:637-49.

11. Kolde R, Laur S, Adler P, Vilo J. Robust rank aggregation for gene list integration and meta-analysis. Bioinformatics. 2012;28:573-80.

12. Bioinformatics RKSLPA. VJJ: Robust rank aggregation for gene list integration and meta-analysis. 2012, 28:573-580.

13. Fan Y, Siklenka K, Arora SK, Ribeiro P, Kimmins S, Xia J. miRNet - dissecting miRNA-target interactions and functional associations through network-based visual analysis. Nucleic Acids Res. 2016;44:W135-41.

14. AL C-G, M S-G IB, JM MTIARF, MMDDG, M S-d-I-T, Respirology J: Effect of age on the cardiovascular remodelling induced by chronic intermittent hypoxia as a murine model of sleep apnoea. 2020, 25:312-320.

15. Sandoval J, Del Valle-Mondragon L, Masso F, Zayas N, Pulido T, Teijeiro R, Gonzalez-Pacheco H, Olmedo-Ocampo R, Sisniega C, Paez-Arenas A, et al: Angiotensin Converting Enzyme 2 and Angiotensin (1-7) axis in Pulmonary Arterial Hypertension. Eur Respir J 2020.

16. H Z YG. Y S, N Z, physiology WXJJoc: miR-181a/b-5p ameliorates inflammatory response in monocrotaline-induced pulmonary arterial hypertension by targeting endocan. 2020, 235:4422-4433.

17. W Z TWXZRLXLJWXZ. Y L, X H, W L, et al: Integrated bioinformatic analysis reveals YWHAB as a novel diagnostic biomarker for idiopathic pulmonary arterial hypertension. 2019, 234:6449-6462.

18. X S. G C, L J, S L, X W, J Z, M W, W L, cellular LCJJo, medicine m: Analysing the relationship between IncRNA and proteincoding gene and the role of IncRNA as ceRNA in pulmonary fibrosis. 2014, 18:991-1003.

19. MZ Z, HD Q, XJ Y, RH Z, LZ C. QS F, medicine ZYJJot: Haplotype of gene Nedd4 binding protein 2 associated with sporadic nasopharyngeal carcinoma in the Southern Chinese population. 2007, 5:36.

20. MC G-P MO, Cellular TDPJ. CMLS mls: BMP type II receptor as a therapeutic target in pulmonary arterial hypertension. 2017, 74:2979-2995.

21. Kumar R, Mickael C, Chabon J, Gebreab L, Rutebemberwa A, Garcia AR, Koyanagi DE, Sanders L, Gandjeva A, Kearns MT, et al. The Causal Role of IL-4 and IL-13 in Schistosoma mansoni Pulmonary Hypertension. Am J Respir Crit Care Med. 2015;192:998-1008. 
22. vdS LSM, vS CRAM, dL A, vR MHSTJ, HJ RS. B, et al: Stiffness-Induced Endothelial DLC-1 Expression Forces Leukocyte Spreading through Stabilization of the ICAM-1 Adhesome. 2018, 24:3115-3124.

23. K J, LM M, AC JH, H T-P MOBWB. D K, K S, W K, et al: Basement Membrane Remodelling Controls Endothelial Function in IPAH. 2020.

24. Kwee BJ, Budina E, Najibi AJ, Mooney DJ. CD4 T-cells regulate angiogenesis and myogenesis. Biomaterials. 2018;178:10921.

25. RT AMGVAL. S, pharmacology RSJBjo: The role of chemokines and chemokine receptors in pulmonary arterial hypertension. 2019.

26. TE S. BK K-H, pharmacology HAJBjo: Inflammasomes: a novel therapeutic target in pulmonary hypertension? 2019, 176:1880-1896.

27. N S, I S, O P, journal WNJTEr: Oxygen sensing and signal transduction in hypoxic pulmonary vasoconstriction. 2016, 47:288-303.

28. JK LHTOAG, Chest HNL. KHJ: Does circulating IL-17 identify a subset of patients with idiopathic pulmonary arterial hypertension? 2015, 148:e131-e132.

29. T LWJLWWXQYWBTHDJWWN, Thorax Y. WCJ: Targeting IL-17 attenuates hypoxia-induced pulmonary hypertension through downregulation of $\beta$-catenin. 2019, 74:564-578.

30. C LL, Y J-H WIKK, Hypertension GSJ. Inhibition of nuclear factor-KB in the lungs prevents monocrotaline-induced pulmonary hypertension in mice. 2014, 63:1260-1269.

31. K RK, ME A-M SNKHKDS, MAH S. J O, T S, S S, et al: Identification of Celastramycin as a Novel Therapeutic Agent for Pulmonary Arterial Hypertension. 2019, 125:309-327.

32. W HTIMBAF, MN T, NHT HMB. TH, CA B, IH H, Allergy vdBMJ: MiR-31-5p: A shared regulator of chronic mucus hypersecretion in asthma and chronic obstructive pulmonary disease. 2020, 75:703-706.

33. Q JLJS. L, M Q, M Z, cancer JFJT: MicroRNA-based biomarkers for diagnosis of non-small cell lung cancer (NSCLC). 2020, $11: 762-768$.

34. C YLYLRL, X YJZDH. Z, OncoTargets ZJJ, therapy: Four-miRNA signature as a prognostic tool for lung adenocarcinoma. 2018, 11:29-36.

35. PY ADDWLT, KJ D-S X, E KHC, CCT AMMFP, Physiology H. ASJC: Mitochondria in the Pulmonary Vasculature in Health and Disease: Oxygen-Sensing, Metabolism, and Dynamics. 2020, 10:713-765.

36. JT Z, FK HQ, DD MC,JS, SY Z, XF L, JT LJQ, BY L. J, et al: Plasma extracellular vesicle microRNAs for pulmonary groundglass nodules. 2019, 8:1663666.

37. K I SS. H N, H N, S O, medicine IYJJoc: EGFRUnique MicroRNA and mRNA Interactions in -Mutated Lung Adenocarcinoma. $2018,7$.

38. Y OI SN. A A, biology GDBJOajoi: Meta-microRNA Biomarker Signatures to Classify Breast Cancer Subtypes. 2018, 22:709716.

39. XH L, Z X, Neoplasma GCJ. MiRNA-500a-3p inhibits cell proliferation and invasion by targeting lymphocyte antigen 6 complex locus K (LY6K) in human non-small cell lung cancer. 2018, 65:673-682.

40. C J. J L, B L, M X, W W, X X, X W, experimental KMJJo, CR ccr: miR-500a-3p promotes cancer stem cells properties via STAT3 pathway in human hepatocellular carcinoma. 2017, 36:99.

\section{Figures}




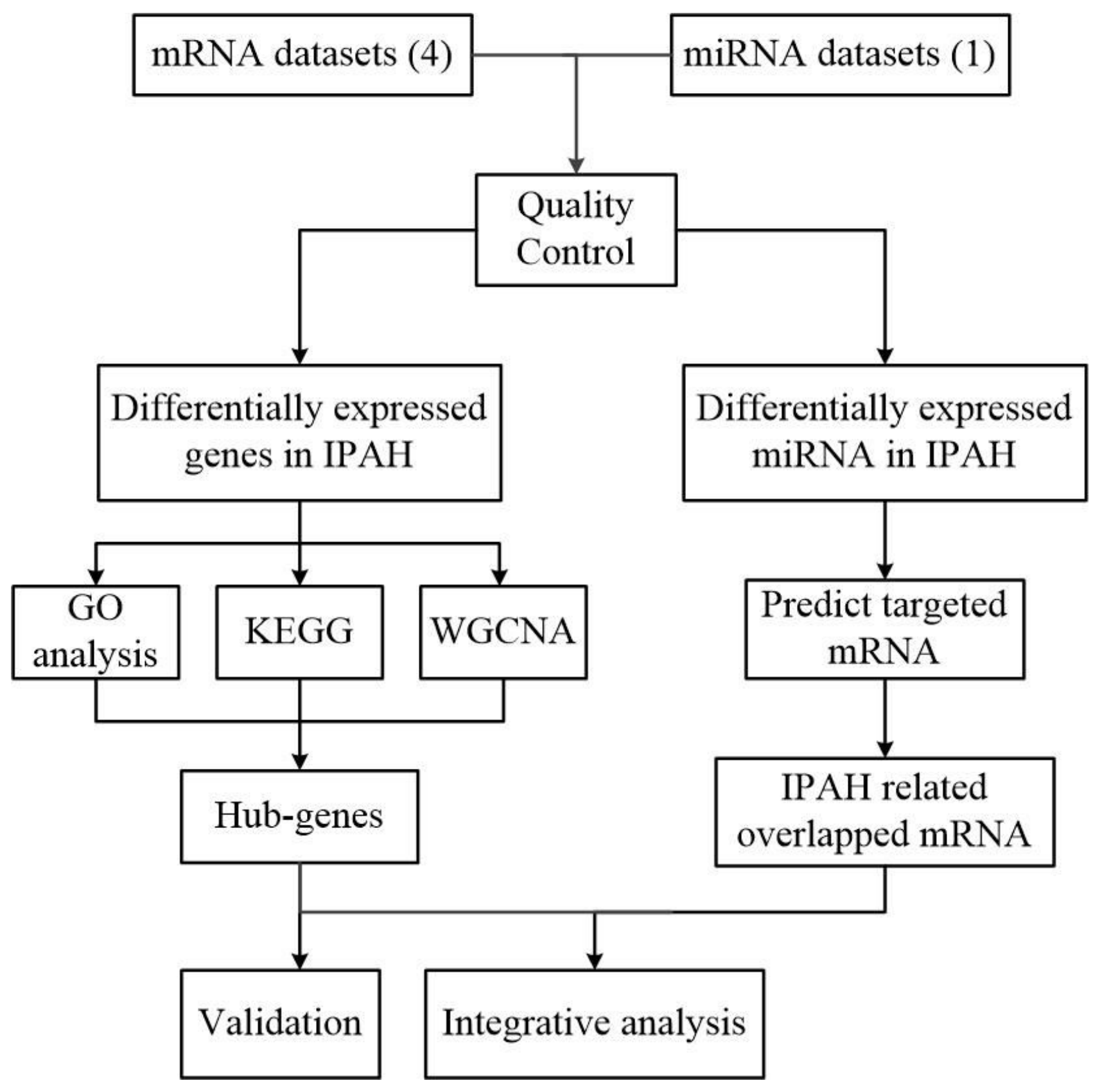

Figure 1

Flowchart of the bioinformatic analysis. miRNA, microRNA; RRA, the Robust Rank Aggregating; IPAH, idiopathic pulmonary arterial hypertension; GO, Gene Ontology; KEGG, Kyoto Encyclopedia of Genes and Genomes; WGCNA, the weighted gene coexpression network analysis. 


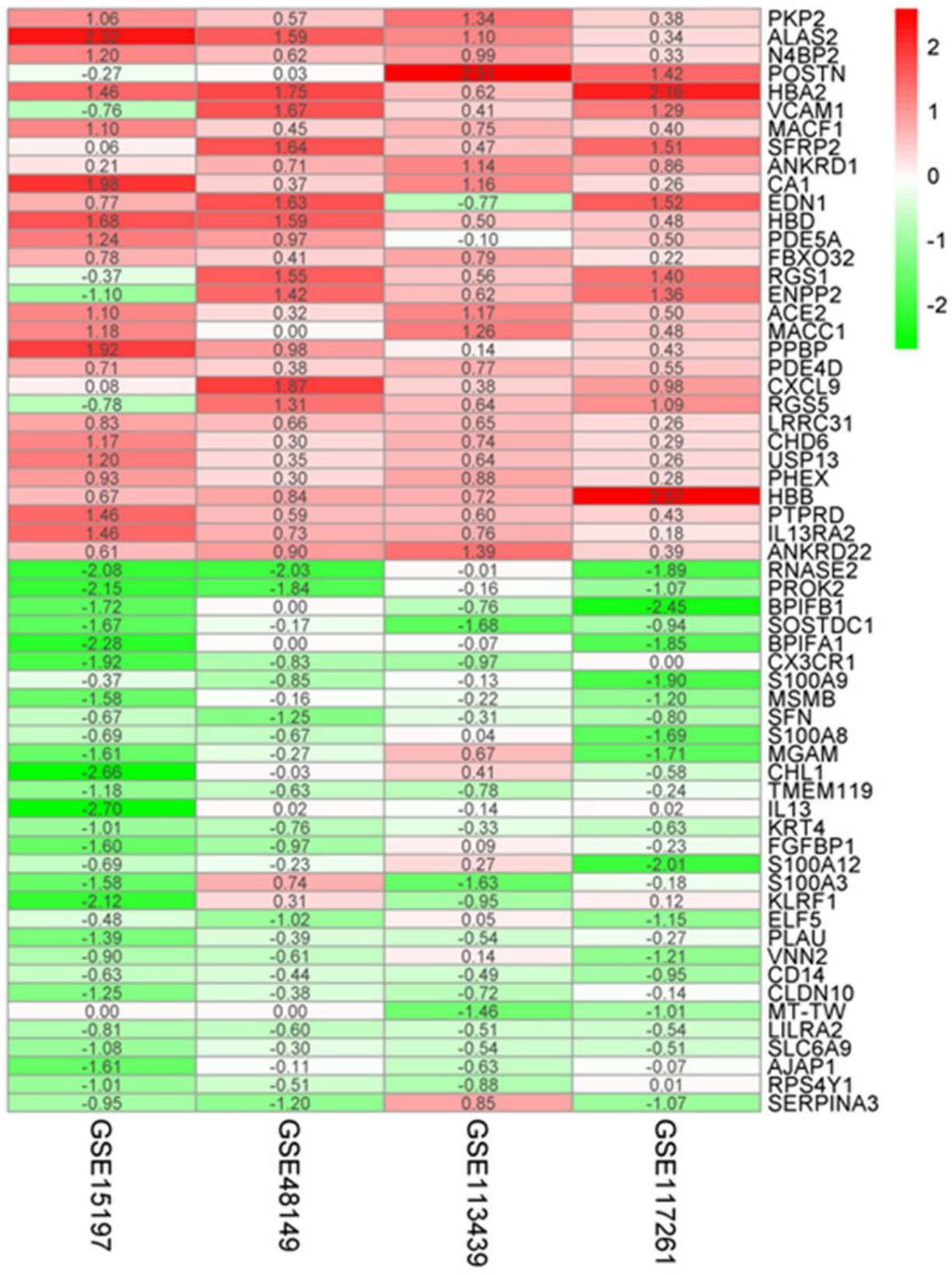

Figure 2

Robust DEGs identified by RRA analysis. Heatmap of the four datasets showing the top 30 up-regulated and 30 down-regulated DEGs. The horizontal axis indicates the gene name, and the vertical axis represents a dataset. Red indicates that the gene is upregulation in the IPAH patients compared with the controls, and the green represents down-regulation. The number in a cell indicates the logFC of each gene in a dataset. DEG, differentially expressed gene; RRA: robust rank aggregation. 
A

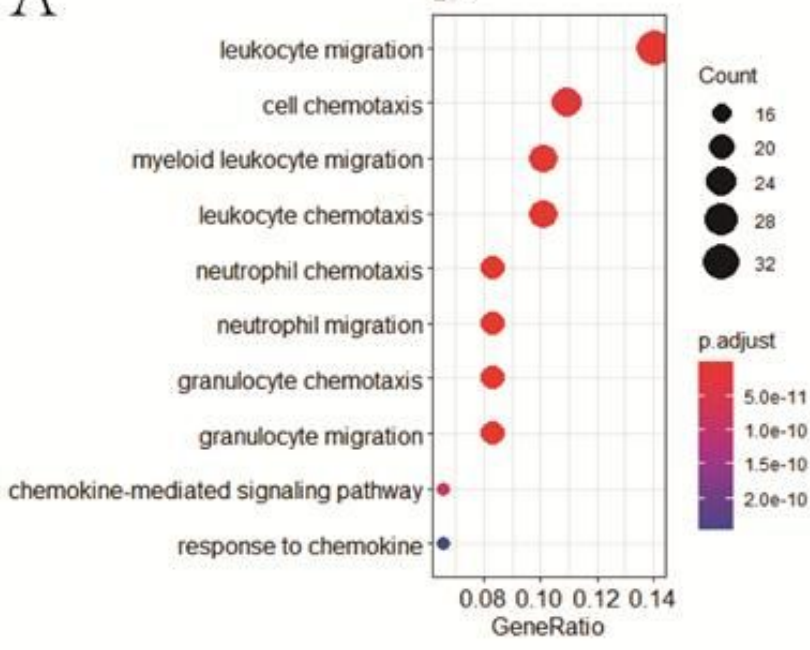

C

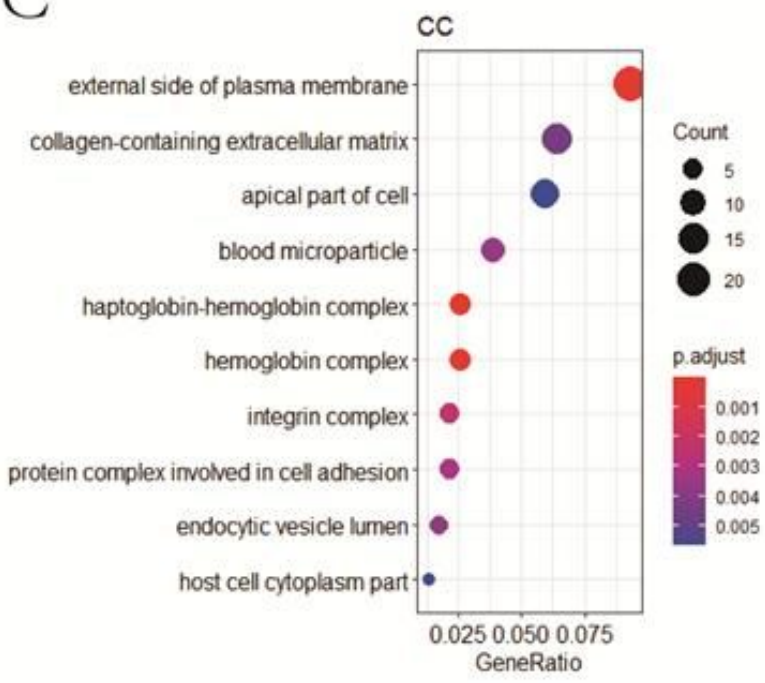

B

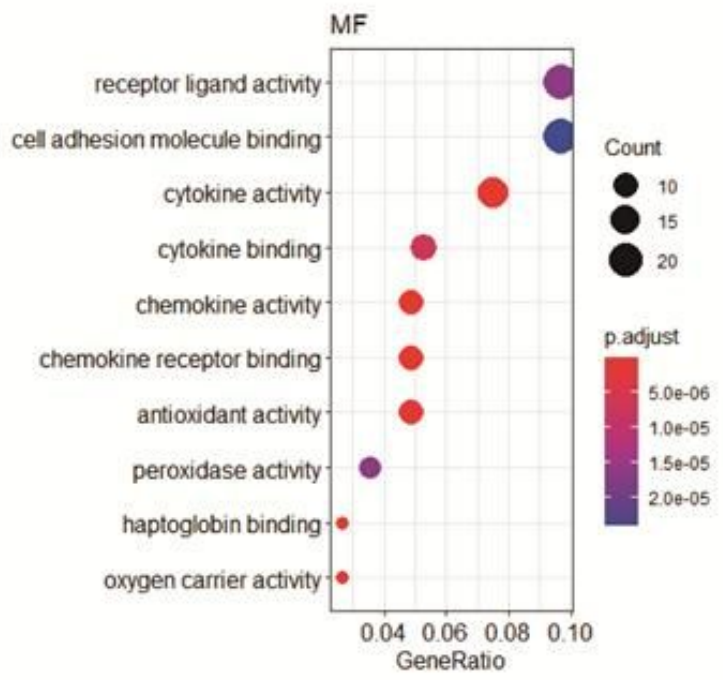

D

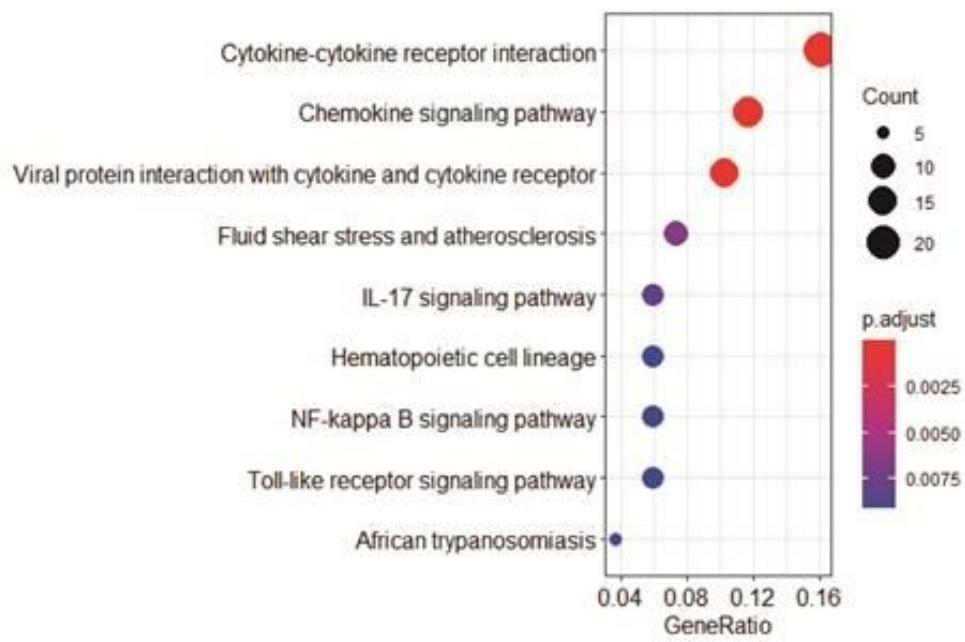

\section{Figure 3}

GO, KEGG pathway enrichment the robust DEGs in IPAH. (A) Top 10 biological processes. (B) Top 10 molecular functions. (C) Top 10 cellular components. (D) Top10 of the KEGG pathway. 
A

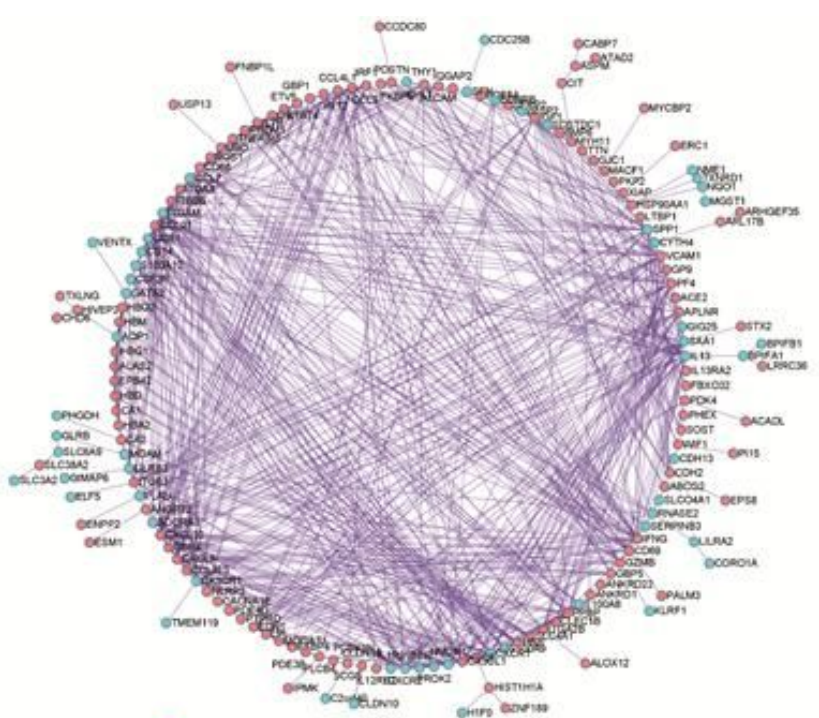

B

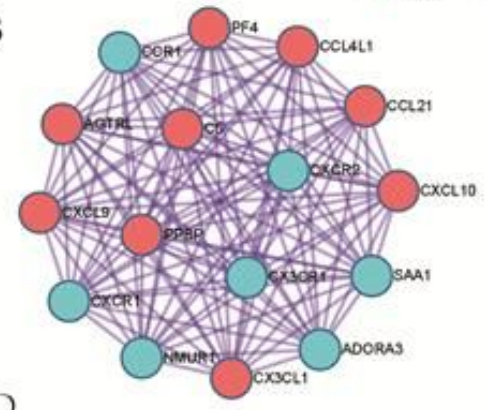

C

D
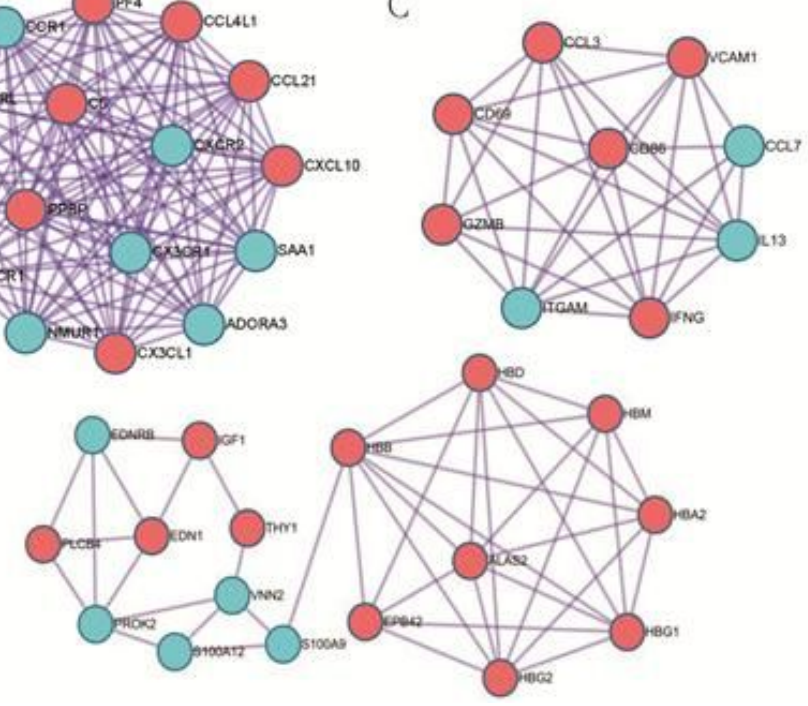

Figure 4

The PPI network of the robust DEGs. (A) PPI network of up-regulated and downregulated significant genes. (B, C, D) The most significant modules identified through MCODE in Cytoscape software. 

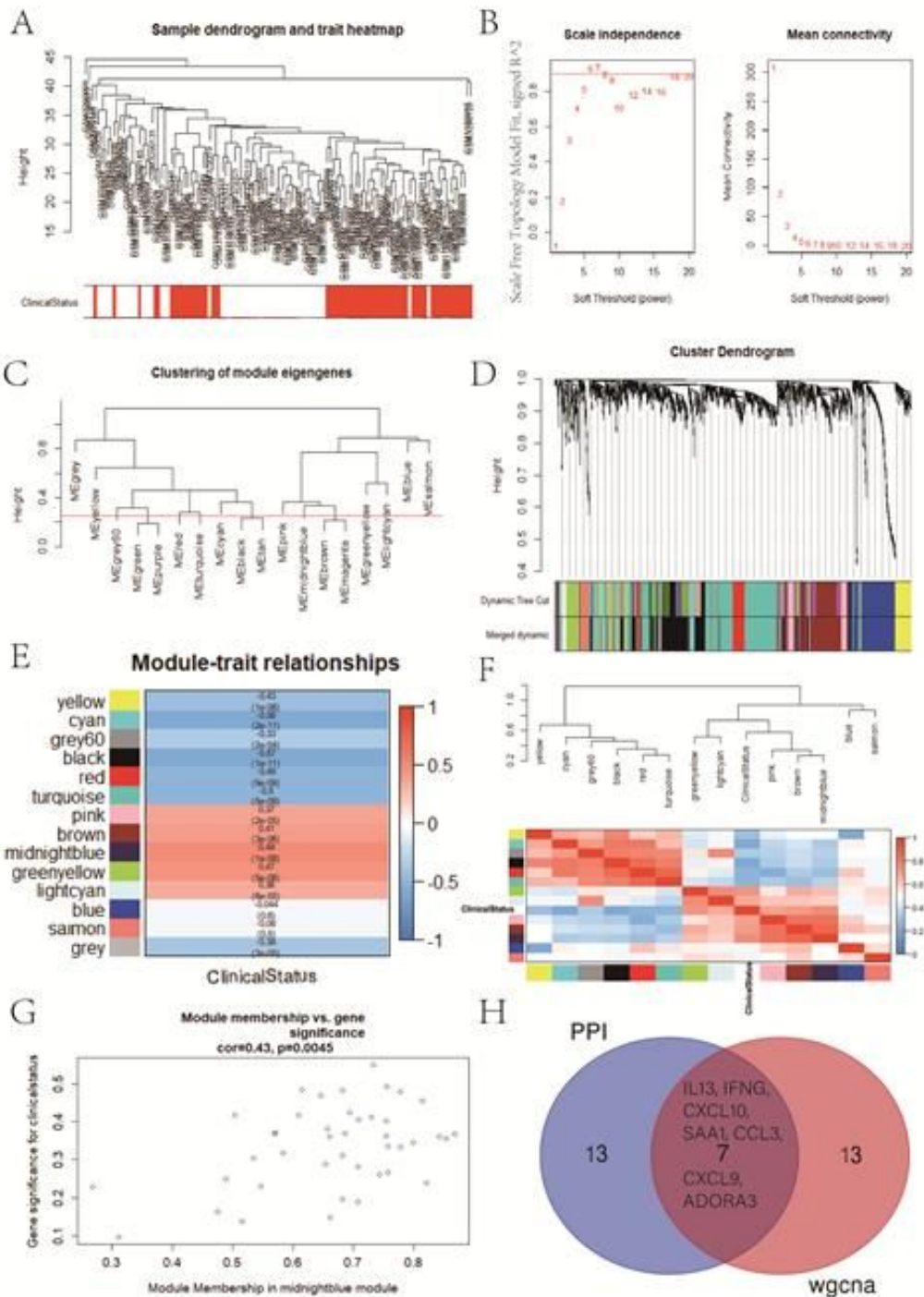

$\mathrm{F}$

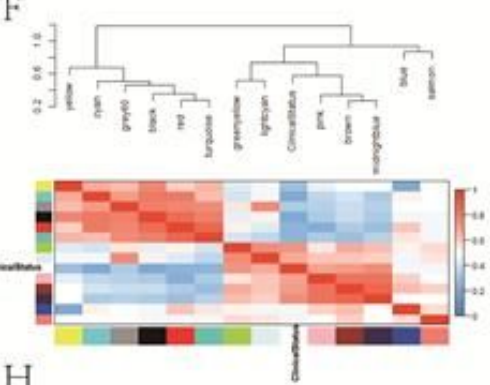

$\mathrm{H}$

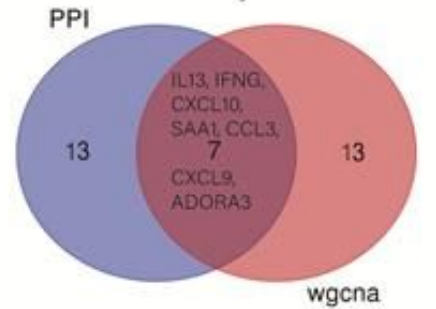

\section{Figure 5}

Identification of crucial modules associated with IPAH by WGCNA. (A) Clustering dendrograms of genes from bath-normalized four datasets. In the column of clinical status, red indicates IPAH and white means the control. (B) The scale-free fit index (left) and the mean connectivity (right) for various soft-thresholding powers. (C) Clustering of module eigengenes. The cut height (red line) was 0.25. (D) Dendrogram of the DEGs clustered based on a dissimilarity measure (1-TOM). (E) Heatmap showing the relationship between module eigengenes and clinical status. The numbers in each cell means the correlation coefficient and Pvalue. (F) Cluster analysis and heatmap of the genes in different modules. Red means a positive correlation, and blue indicates a negative correlation. $(\mathrm{G})$ Scatter plot of module eigengenes in the midnight-blue module. $(\mathrm{H})$ Venn diagram. The overlap of top20 genes in the midnight-blue module of WGCNA and top20 hub genes of PPI analysis. 
A

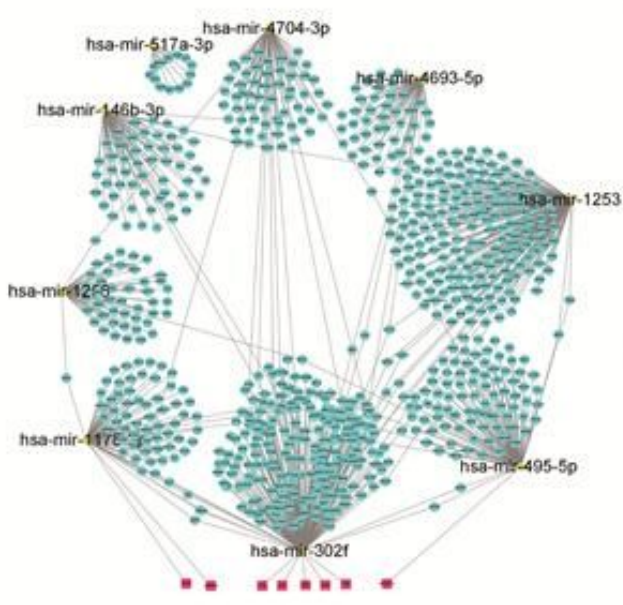

D

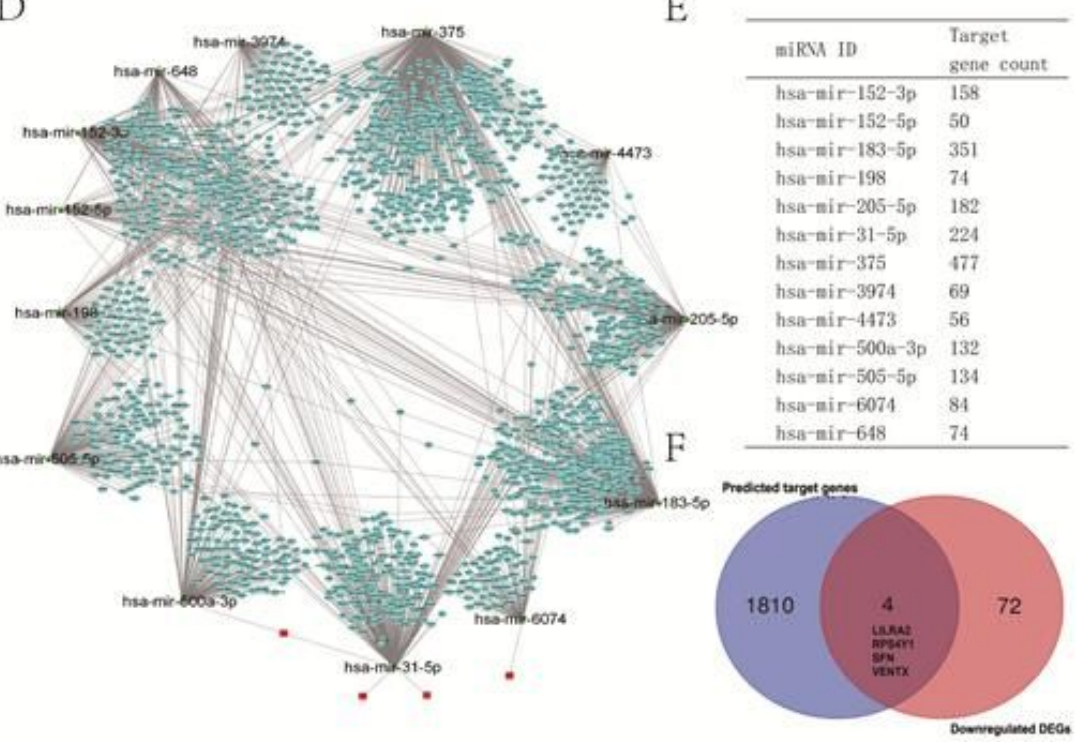

B

$\begin{array}{ll}\text { miRNA ID } & \text { Target } \\ \text { gene cotht }\end{array}$

hsa-air-517a-3p 13

hsa-mir-146b-3p 56

hsa-mir-1178-3p 69

hsu-nir-1253 195

hss-nir-1256 46

hsa-nir-495-5p 92

hsa-nir-302f 227

hsa-nir-4693-5p 49

hsu-nir-4704-3p 66

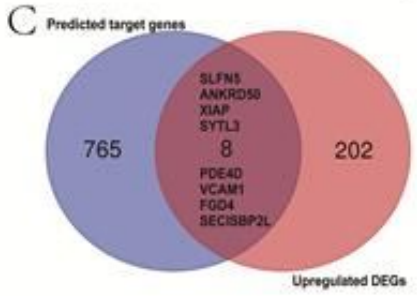

E

\begin{tabular}{ll} 
miRSA ID & $\begin{array}{l}\text { Target } \\
\text { gene count }\end{array}$ \\
\hline hsa-nir-152-3p & 158
\end{tabular}

hsa-nir-152-5p 50

hsa-mir-183-5p 35

mir-198 74

sa-nir-500a-3p 13:

\section{Figure 6}

Potential target genes of DE-miRNAs predicted by miRNet database. (A) The network of downregulated DE-miRNAs and predicted target genes. (B) Predicted target gene count for each downregulated DE-miRNAs. (C) The Venn gram of predicted upregulated target genes and upregulated DEGs from RRA analysis. (D) The network of upregulated DE-miRNAs and predicted target genes. (E) Predicted target gene count for each upregulated DE-miRNAs. (F) The Venn gram of predicted downregulated target genes and downregulated DEGs from RRA analysis. 


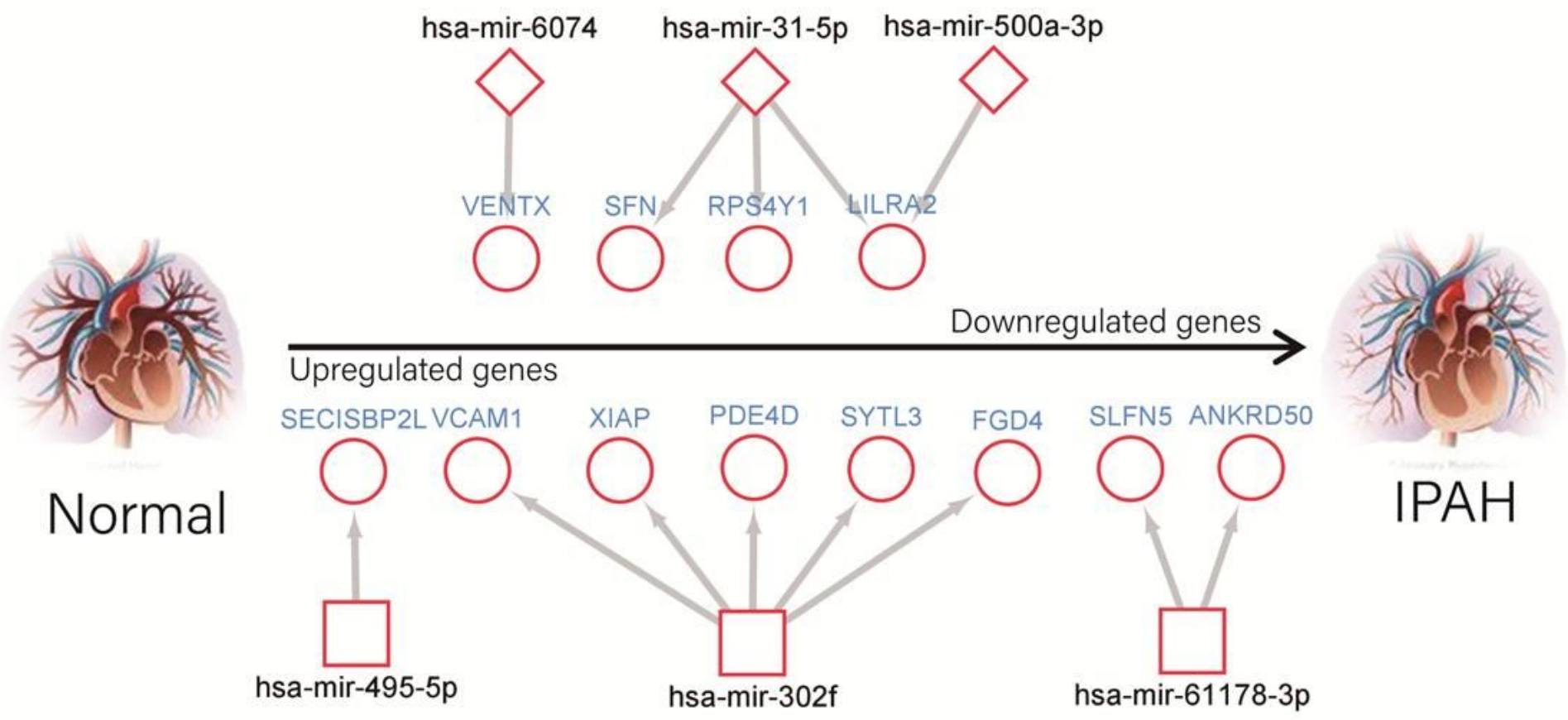

Figure 7

An interactive network of overlapped genes and the upstream miRNAs. 
A
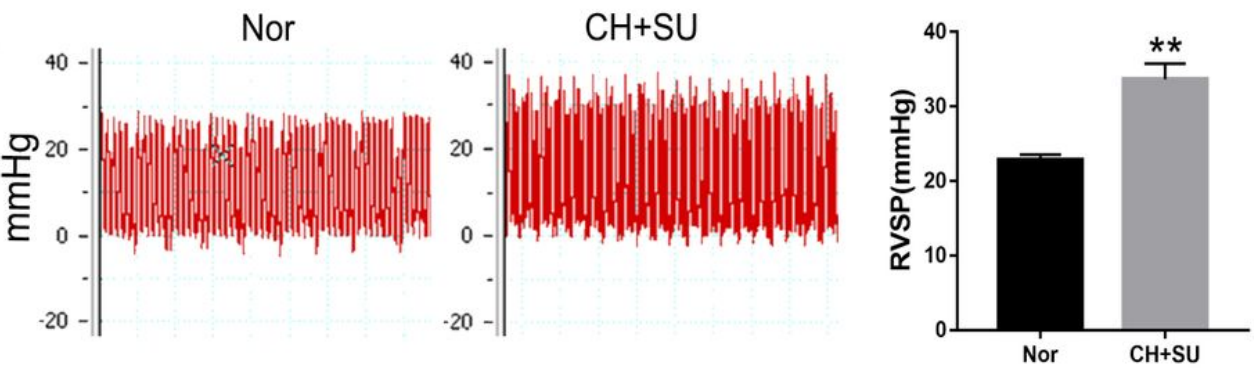

$\mathrm{B}$

C

Nor

$\mathrm{CH}+\mathrm{SU}$
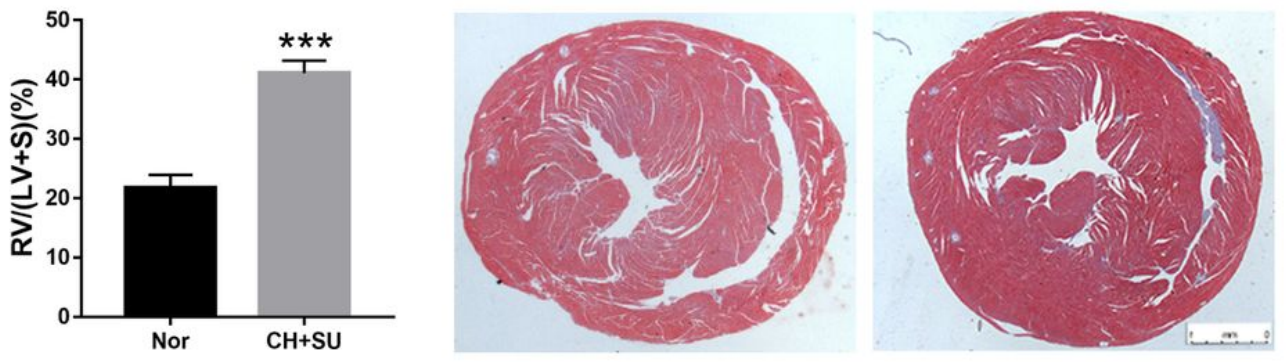

D
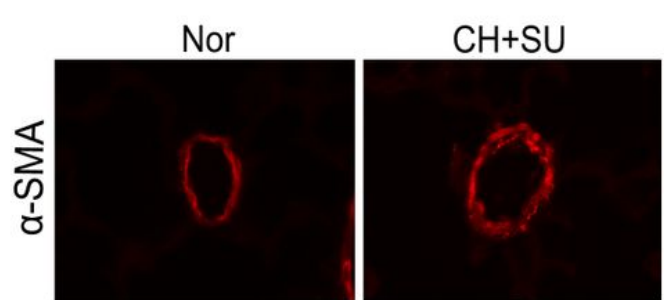

E Nor

$\mathrm{CH}+\mathrm{SU}$
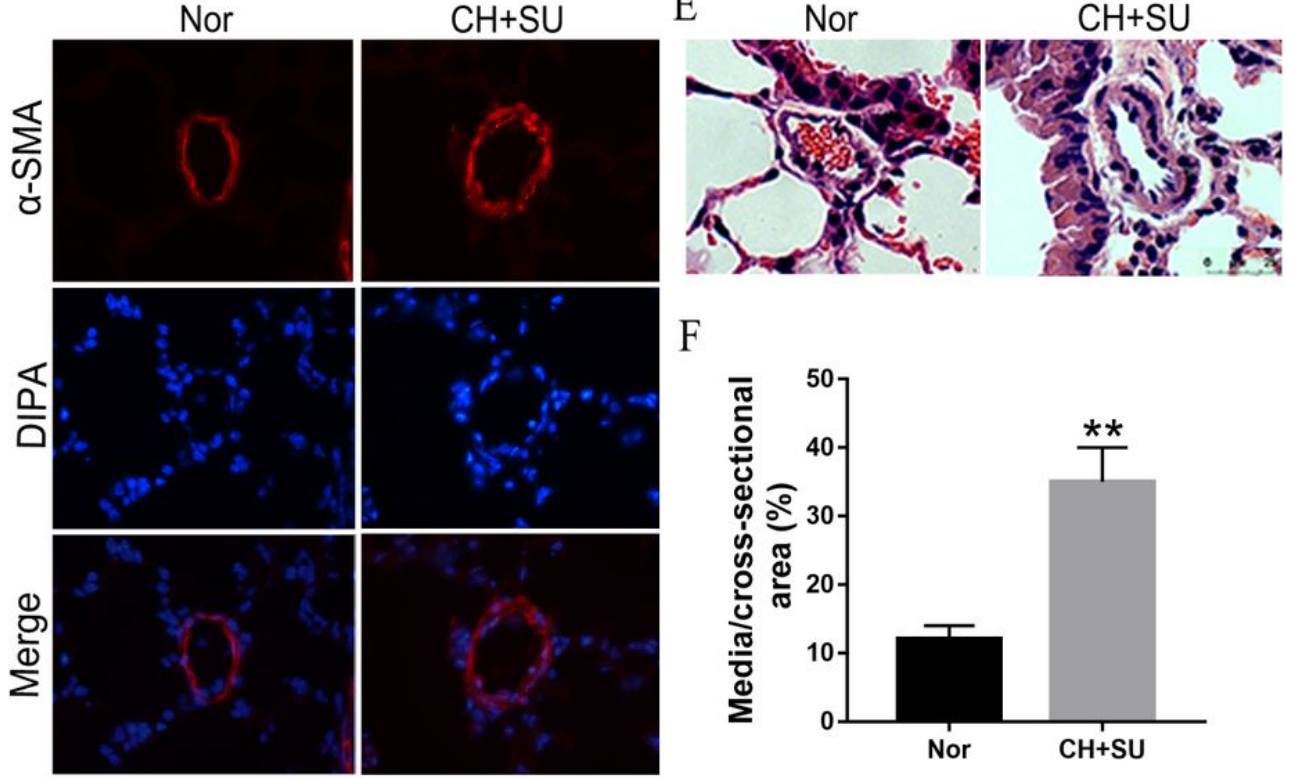

$\mathrm{F}$

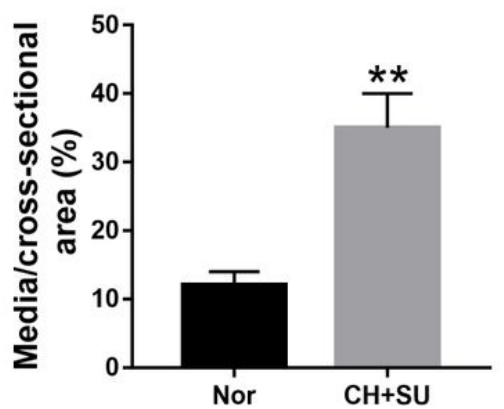

\section{Figure 8}

PAH mice model. (A) Representative tracing of RVSP in mice after CH+SU or Nor treatment (left) and the mean values of RVSP in the two groups. (B) Fulton index (RV/LV+S) in mice after $\mathrm{CH}+\mathrm{SU}$ or Nor treatment. (C) Representative Masson stain images of the hearts from $\mathrm{CH}+\mathrm{SU}$ or Nor mice. (D) Representative a-SMA immunostaining images of lung sections from $\mathrm{CH}+\mathrm{SU}$ or $\mathrm{Nor}$ mice. Bar, $25 \mu \mathrm{m}$. (E) Representative images of H\&E staining of lung sections from CH+SU or Nor mice. Bar, $25 \mu \mathrm{m}$. (F) The ratio of pulmonary arterial medial thickness to total vessel size for the $\mathrm{CH}+\mathrm{SU}$ or Nor mice. 
A

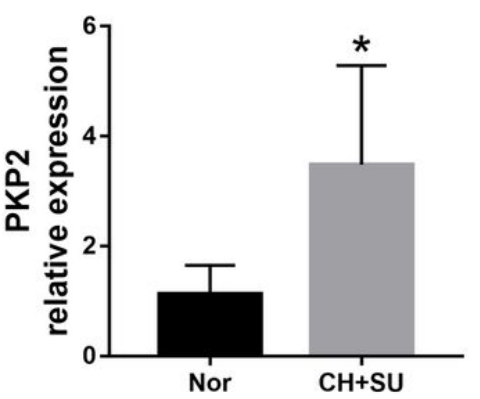

C

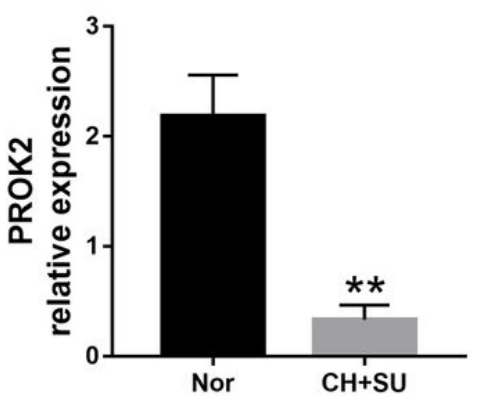

E

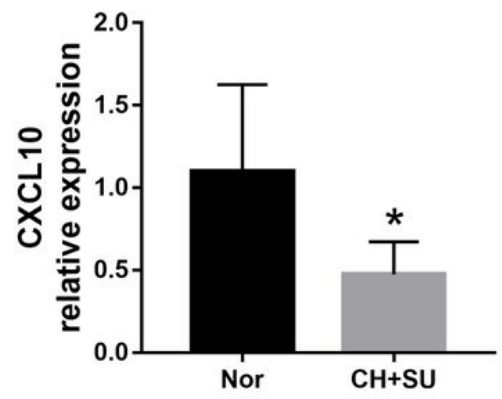

G

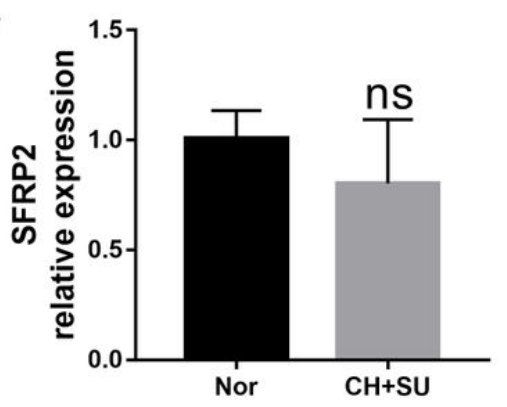

B

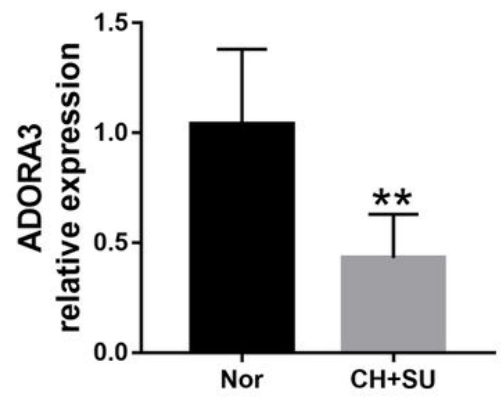

D

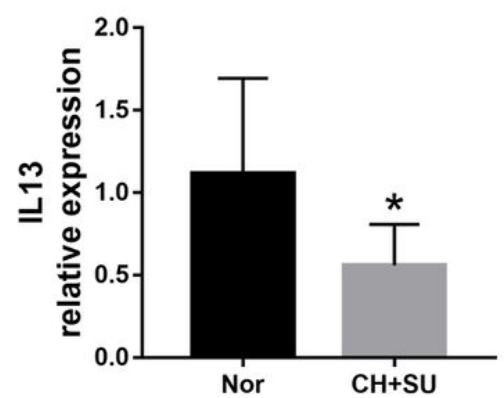

F

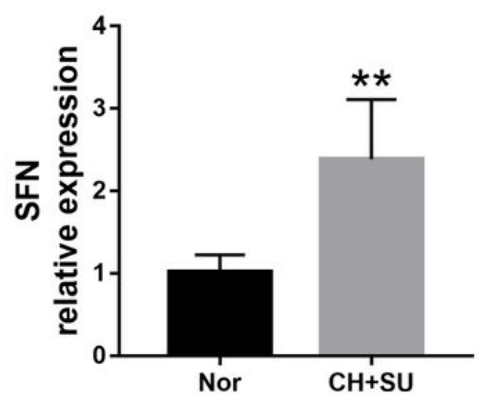

Figure 9

Validation of seven differently expressed key genes through RT-PCR in the lungs from CH+SU or Nor mice. Expression of PKP2 (A), ADORA3 (B), PROK2 (C), IL-13 (D), CXCL10 (E), SFN (F), and SFRP2 (G) in CH+SU mice compared with Nor controls. PKP2, plakophilin-2; ADORA3, adenosine A3 receptor; PROK2,prokineticin 2; IL-13, interleukin-13; SFN, stratifin; SFRP2, secreted frizzledrelated proteins. $\mathrm{n}=6$ in each group. Values are given as the mean \pm SEM.

\section{Supplementary Files}

This is a list of supplementary files associated with this preprint. Click to download.

- supplement.docx 